\title{
Characterization of Helium Ion Implantation Damage in Metallic Multilayers
}

\author{
A. Misra, D. Bhattacharyya, Q. Wei, and M. Nastasi. \\ Los Alamos National Laboratory, Los Alamos, NM 87545.
}

Interfaces act as obstacles to slip and sinks for radiation-induced defects. Hence, nanolayered composites provide over an order of magnitude increase in strength and enhanced radiation damage tolerance compared to bulk materials [1]. Here we use model fcc-bcc systems such as $\mathrm{Cu}-\mathrm{Nb}$ and $\mathrm{V}$ Ag to experimentally measure the critical concentration of helium at which bubbles are detected in de-focused transmission electron microscopy (TEM) images.

Figure 1 shows an under-focused bright-field TEM image from a $\mathrm{Cu}-\mathrm{Nb}$ multilayer with a nominal layer thickness of $6 \mathrm{~nm}$ implanted with $35 \mathrm{keV} \mathrm{He}+$ ions to a dose of $1 \times 10^{17} / \mathrm{cm}^{2}$. He bubbles appear as bright dots. The measured size of these bright dots when plotted as a function of defocus allowed for the estimation of bubble size $(\approx 1 \mathrm{~nm})$ at zero defocus. The concentration of helium as a function of depth was measured using either secondary ion mass spectroscopy or ion beam techniques such as nuclear reaction analysis (NRA). From Figure 1, the depth below which the bubbles are not observed gives (using the NRA measured helium concentration profile) the critical concentration of helium at which bubbles are precipitated at interfaces. For a $6 \mathrm{~nm}$ layer thickness multilayer, this critical concentration was found to be approximately 2 atomic\%, significantly higher than bulk metals where the helium solubility is negligible. In single layer $\mathrm{Cu}$ film (Figure 2), implanted under the same conditions as Figure 1, helium bubbles are detected right at the film surface. The high helium concentration for bubble nucleation in multilayers is due to the high solubility of helium at $\mathrm{Cu}-\mathrm{Nb}$ interfaces.

In a different experiment [2], $35 \mathrm{keV} \mathrm{He}^{+}$ions were implanted at $490{ }^{\circ} \mathrm{C}$ to a total dose of $1 \times 10^{17}$ $/ \mathrm{cm}^{-2}$ in two different multilayers: $120 \mathrm{~nm}$ and $4 \mathrm{~nm}$. In the $120 \mathrm{~nm}$ samples, large faceted bubbles, most exceeding $20 \mathrm{~nm}$ in diameter, were observed in the $\mathrm{Cu}$ layers. However, in the $\mathrm{Nb}$ layers the bubbles remained small, approximately 1-2 $\mathrm{nm}$ in diameter. In the $4 \mathrm{~nm}$ samples, the Nb layers still showed the 1-2 $\mathrm{nm}$ size bubbles but the size of the bubbles in the $\mathrm{Cu}$ layer was limited by the layer thickness of $4 \mathrm{~nm}$. So, there emerges the very interesting behavior that, bubbles in the $\mathrm{Cu}$ layer grow to the thickness of the layer and stop at the interface.

The $\mathrm{Cu}-\mathrm{Nb}$ system predominantly exhibits Kurdjumov-Sachs (K-S) orientation relationship, $\{111\} \mathrm{fcc} / /\{110\} \mathrm{bcc},<110>\mathrm{fcc} / /<111>\mathrm{bcc}$. In order to understand the effects of interface geometry, another fcc-bcc system V-Ag was studied. In V-Ag, in addition to $\mathrm{K}-\mathrm{S}$, other orientation relationships such as Nishiyama-Wasserman, Bain and Pitsch were observed. Helium solubility at these different interfaces is measured and interpreted in terms of atomic structure of the interface.

References

[1] A. Misra, et al., JOM, 59 (2007) 62.

[2] K. Hattar, et al., Scripta Materialia, 58 (2008) 541.

[3] This research was supported by the DOE, Office of Science, Office of Basic Energy Sciences. 


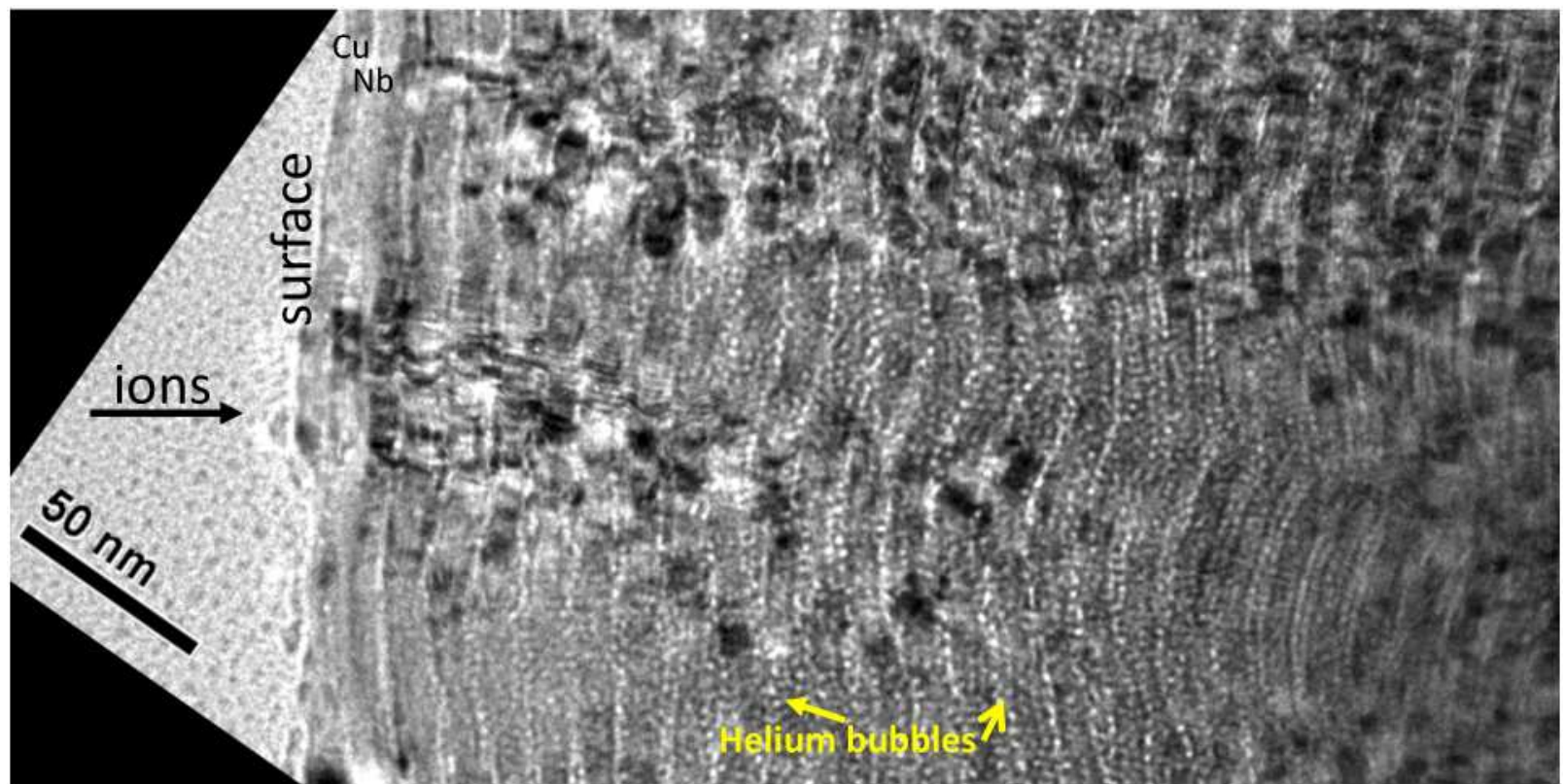

FIG. 1. Under-focused bright field TEM micrograph showing helium bubbles as bright dots in $\mathrm{Cu} / \mathrm{Nb}$ multilayer.

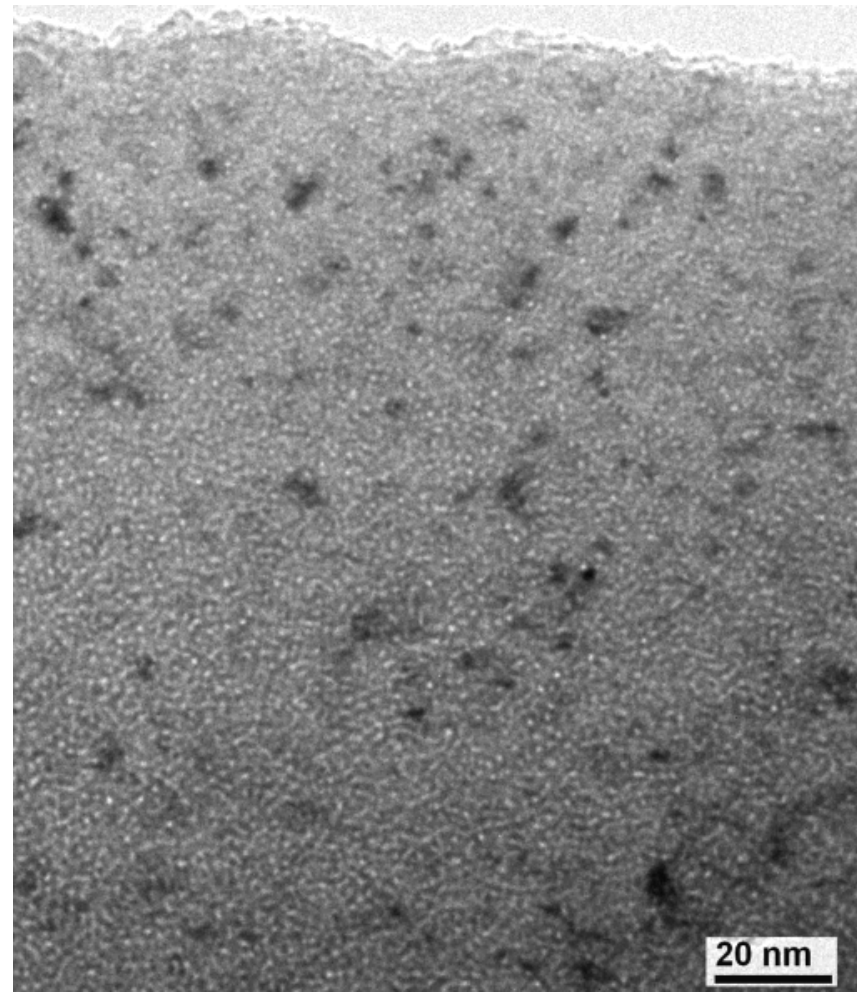

FIG. 2. Under-focused bright field TEM micrograph showing helium bubbles as bright dots in a $\mathrm{Cu}$ film. Note that bubbles are observed very close to the surface where the implanted helium concentration is low. 